\title{
Éditorial
}

\section{Y-a-pas de doute ! Avatar subséquent du principe de précaution?}

Cette expression triviale semble bien avoir contaminé nos élites. Il suffit de quelques exemples - amiante, affaire du sang contaminé, canicule, vache folle (encéphalite spongiforme bovine), grippe aviaire (grippe A- $\mathrm{H}_{5} \mathrm{~N}_{1}$ ) grippe porcine (grippe $\mathrm{A}-\mathrm{H}_{1} \mathrm{~N}_{1}$ ), GIEC (Groupement Intergouvernemental sur l'Evolution du Climat) - pour être convaincu de cette nouvelle pandémie : le doute n'existe plus guère, il peut même être considéré comme une notion subversive ou assimilée à une attitude rebelle. Dans tous ces exemples, on retrouve les mêmes fauteurs de troubles, un groupe d'experts, que certains éminents professeurs ont qualifié d'« experts de pacotille », d'« experts en chambre »..., qui rédigent un rapport quelquefois volumineux (3000 pages pour le rapport initial 2007 du GIEC), souvent peu lu, résumé en quelques directives proposées aux décideurs politiques. Selon les cas, les mesures tardent à se mettre en place (amiante, affaire du sang contaminé, canicule) ou sont démesurées et coûteuses (vache folle, grippes aviaire et porcine).

Tous les politiques n'ont pas le même comportement désinvolte que Mme Georgina Dufoix lors du procès du sang contaminé (« responsable mais pas coupable ») : en général, ils suivent l'avis des experts, quelquefois un peu aveuglément comme pour la grippe A-H1N1. Dans les mesures prises pour ce qui devait être une pandémie, on retrouve tous les signes d'un dysfonctionnement qui a conduit à l'échec de la campagne de vaccination : manque de transparence dans la formation et le fonctionnement du groupe d'experts, secret des discussions, manipulations sur la base d'un catastrophisme éclairé, présence de lobbies, organisation quasi militaire de la campagne de vaccination, esprit de propagande, mesures coercitives... Cette façon de procéder a conduit à une couverture vaccinale décevante et à l'inutilisation de millions de doses de vaccin. Dans la gestion de cette épidémie, on a constaté une absence de dialogue, un manque de confiance, voire un peu de mépris envers les professions de la santé, les enseignants et les parents d'élèves... et l'existence de liens entre les experts du comité pandémie de l'OMS et l'industrie pharmaceutique. À l'heure du bilan on peut se demander avec Gaël Villeneuve (Vaccinés à la norme. Esprit, février 2010) si cette crise n'est pas le témoignage d'« une situation où ceux qui savent parler aux gestionnaires sont plus écoutés que ceux qui savent de quoi ils parlent ».

Bien des groupes d'experts peuvent connaitre ce dysfonctionnement. L'exemple le plus caricatural semble être celui du GIEC. Cet organisme créé en 1985, placé sous l'égide de l'0NU en 1993, a reçu conjointement avec Albert A. Gore Jr - Vice-président des USA sous la présidence de Bill Clinton, lequel refusa de signer les accords de Kyoto - le prix Nobel de la Paix(?) en 2007. Fonctionnant également sur le même mode (manque de transparence, refus de communiquer les sources, conflits d'intérêts...), le GIEC fait l'objet de vives critiques depuis que des erreurs grossières ont été découvertes dans le rapport 2007 (date de la fonte possible des glaciers de l'Himalaya, perte de $40 \%$ de la forêt amazonienne, submersion de $55 \%$ des Pays-Bas ...). Les « climatoseptiques » sont devenus pour l'occasion des « giéco-septiques», affirmant que «le débat scientifique est devenu quasi religieux » (Vincent Courtillod), que le GIEC a abandonné le domaine de la science au profit d'une « croyance collective » (Serge Galam); ce dernier conclut qu'« il faut se méfier des scientifiques coalisés ». Effectivement au fil des années, plusieurs membres éminents du GIEC ont été amenés à démissionner parce qu'ils refusaient de cautionner certaines conclusions qui avaient été adoptées selon le mode consensus d'experts, c'est-à-dire après un vote! C'est le cas par exemple de Charles Landsea qui a démissionné pour «ne pas contribuer à un processus aux objectifs préconçus et scientifiquement non fondés ».

Pour Mike Ulme et d'autres, le GIEC a dépassé sa date de péremption et nécessiterait une réforme profonde d'autant que le Président Rajendra Pachauri est soupçonné de conflits d'intérêts financiers. Cet Indien de 69 ans, Président du GIEC depuis 2002, a résisté à toutes les accusations mais il vient de commettre une dernière imprudence (ou c'est peut-être une provocation) qui lui sera probablement fatale, mais qui discréditera un peu plus le GIEC. Il vient de publier un roman intitulé Retour à Almora que le Time of India a qualifié de « roman de gare de la spiritualité ». En cette période de fortes turbulences dans le milieu climatologique, le Président du GIEC aurait dû avoir d'autres préoccupations? Et, comme pour provoquer l'orage, il a fait éditer son livre par 
Mukesh Ambani - Président de Reliance Industries et première fortune indienne dans le secteur du pétrole et du gaz - et fait financer les festivités de lancement de son livre par la firme pétrolière BP! Par ailleurs, ces deux firmes sont clientes du cabinet de conseil Pachauri, Teri. ...!

Revenons au domaine de la santé. Pendant que l'on assistait d'un côté de la disparition des notes et de la distribution des prix dans l'enseignement secondaire, et au baccalauréat pour tous ou presque, on vit de l'autre côté apparaître de nombreux classements concernant les villes, les restaurants, les vins, les lycées. .. les hôpitaux et leur différents services. Depuis quelques années, l'Université de Shanghai propose un classement mondial des universités. Celles qui sont bien notées ne manquent pas d'en faire état et de l'afficher sans se soucier de la méthodologie qui a permis d'établir le classement. À contrario, celles qui sont mal classées on tendance à dénigrer le principe et la méthode.

Que se passe-t-il dans le domaine de la santé. Les épisodes de la grippe aviaire et de la grippe porcine représentent-ils seulement des épiphénomènes ou sont-ils le stigmate d'une société qui refuse d'assumer le doute et le risque? Pour notre profession, l'évaluation est sous-jacente dans de nombreuses démarches : evidence-based medicine (EBM) ou dentistry (EBD), recommandations de bonne pratique, évaluation des pratiques professionnelles, impact factor, index h. . . Certes, l'objectif avoué est louable mais on peut assister très rapidement à une dérive : les recommandations pour la prévention de l'endocardite infectieuse constituent sans doute l'exemple récent le plus criant. Après bien des modifications qui semblaient logiques, comment en est-on arrivé à considérer que le risque d'endocardite est nul lorsqu'il devient très faible? Comment un pays comme la Grande-Bretagne peut-il avoir deux types de recommandations bien différentes : Prophylaxis against infective endocaditis summary of NICE guidance ( $\mathrm{Br}$ Med J 2008;336:770-1) et Guidelines on the prevention, diagnosis, and treatment of infective endocarditis (Eur Heart J 2009;30:2369-413)?

Dans les milieux de l'enseignement universitaire et de la recherche, on assiste à une autre dérive : c'est l'importance donnée à l'impact factor, et maintenant à l'index h (car il a été conçu par Jorge E. Hirsch), pour les nominations et l'obtention de crédits. Parfois, il n'est même plus nécessaire de citer le titre de l'article mais uniquement de fournir son impact factor. . . c'est dire l'intérêt que l'on accorde au travail effectué. L'impact factor est un outil très utile pour ceux qui ont l'esprit bureaucratique et qui croient détenir un moyen d'évaluation simple et fiable. "Peu importe qu'en pratiquant ainsi on juge plus le journal que les articles du chercheur. Peu importe que les facteurs d'impact soient manipulés par les éditeurs et ne reflètent que très approximativement la qualité d'un journal. Peu importe que, dans un même journal, il existe un facteur 100 entre les articles les plus cités et les moins cités ${ }^{1}$. Devant cette approche grossière, certains ont proposé une évaluation plus concrète, mais toujours en faisant appel à des indices bibliométriques. L'index h dépend directement des citations des différents articles publiés par un auteur. "Malheureusement, le postulat qui veut que l'on puisse juger de la qualité d'un chercheur à l'aune de ses citations recueillies n'est guère plus solide que celui basé sur l'impact factor » ${ }^{1}$ En effet, toutes les citations sont comptabilisées : qu'elles soient favorables ou critiques, que ce soit une citation spontanée ou commanditée, voire même une auto-citation personnelle ou à l'intérieur d'un groupe organisé. D'autres questions se posent : comment déterminer qui est le véritable auteur principal, quel est le rôle exact des co-auteurs, comment ne pas pénaliser les jeunes auteurs... Enfin, quelle valeur accorder à ces citations «puisque seuls $10 \%$ à $20 \%$ des articles cités ont été réellement lus par ceux qui les citent " ? $^{1}$

On reste stupéfait en découvrant tant de turpitudes et tant de suffisance, et on se demande si le principe de précaution ne comporte pas des effets indésirables (peut-être collatéraux) qui pourrait inverser le rapport bénéfice/risques. On a assisté à un véritable phénomène d'apostasie : progressivement la culture de l'erreur a remplacé la culture du doute. Pour toute évaluation - y compris celle d'un étudiant - on doit faire preuve de sagesse et avoir une pensée pour Albert Einstein qui fut recalé à son $1^{\text {er }}$ concours d'entrée à l'École Polytechnique de Zürich en 1895, une dizaine d'années avant de commencer à élaborer les théories de la relativité. Ce génie avait aussi quelques qualités humaines et beaucoup d'humour : on doit lui décerner un satisfecit pour la qualité de ses aphorismes. En voici deux que l'on peut méditer selon la situation : "No amount of experimentation can ever prove me right; a single experiment can prove me wrong » par exemple lors d'une bouffée de suffisance, et "Two things are infinite: the universe and human stupidity, and I'm not sure about the universe », là on se gardera de donner un exemple!

\footnotetext{
1 Ségalat L. La science à bout de souffle. Éditions du Seuil, 2009.
} 